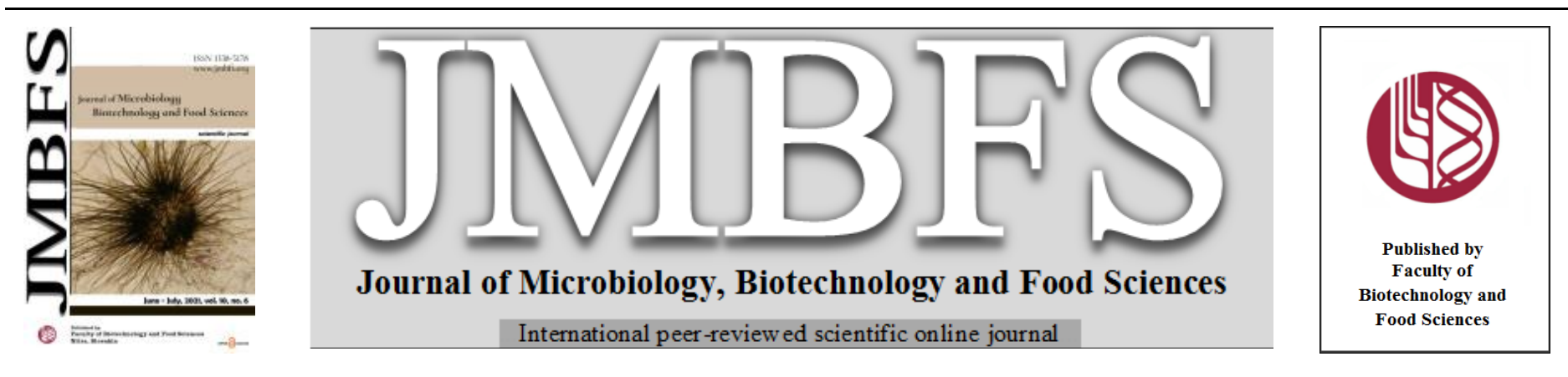

\title{
COMPARATIVE ANALYSIS OF THE EFFECTS OF CURCUMIN AND EPICATECHIN ON THE VITALITY OF ROOSTER SPERMATOZOA
}

\author{
Filip Benko $*^{l}$, Michal Ďuračka ${ }^{I}$, Ján Kováč ${ }^{l}$, Norbert Lukáčl ${ }^{l}$ Eva Tvrdál \\ Address(es): Ing. Filip Benko, \\ ${ }^{1}$ Slovak University of Agriculture, Faculty of Biotechnology and Food Sciences, Department of Animal Physiology, Tr. A. Hlinku 2, 94976 Nitra, Slovak Republic. \\ *Corresponding author: filip.benko276@gmail.com \\ https://doi.org/10.15414/jmbfs.3078
}

\section{ARTICLE INFO}

Received 6. 5. 2020

Revised 7. 5. 2021

Accepted 18. 5. 2021

Published 1. 6. 2021

Regular article

OPEN $\partial_{\text {Access }}$

\begin{abstract}
The objective of our study was to assess the effects of two selected antioxidants (curcumin - CUR and epicatechin - EPI) on the vitality of rooster spermatozoa. We used semen samples from 10 sexually mature Lohmann light roosters, which were collected from a local farm, diluted in PBS and cultured with different concentrations of CUR and EPI $(1,5,10,25,50$ and $100 \mu \mathrm{mol} / \mathrm{L})$. Motility, membrane and acrosome integrity were analysed after 0,2 and $24 \mathrm{~h}$ of in vitro culture. Sperm motility was evaluated by the computer-assisted sperm analysis (CASA). The membrane integrity was determined by a combination of eosin/nigrosin dyes, and the acrosome integrity was detected by fast green/rose bengal dyes. The data were statistically evaluated using One-way ANOVA and the Dunnet's test. The results indicate that after $0 \mathrm{~h}$ of cultivation all selected parameters stay unchanged. After $2 \mathrm{~h}$ significant differences $(\mathrm{P}<0.0001)$ were observed in case of the motility and membrane integrity. Following $24 \mathrm{~h}$ a significant $(\mathrm{P}<0.0001)$ preservation of motility was recorded. The level of membrane damage and acrosome integrity was significantly decreased $(\mathrm{P}<0.01)$. Based on our results we may conclude that protective effects of CUR and EPI on the vitality of rooster spermatozoa were observed already after $2 \mathrm{~h}$. This impact was even more dominant after $24 \mathrm{~h}$. CUR showed better effects at lower concentrations $(1,5$, and $10 \mu \mathrm{mol} / \mathrm{L})$ while EPI at higher doses $(25$ and 50 $\mu \mathrm{mol} / \mathrm{L}$ ). Our findings could contribute to the potential application of these antioxidants in avian reproduction technologies.
\end{abstract}

Keywords: curcumin, epicatechin, rooster, spermatozoa, oxidative stress

\section{INTRODUCTION}

Worldwide poultry farming is focused mainly to produce high-quality eggs and meat. The economic and nutritional demands of meat production require many poultry breeds in confinement during a short period of time. Poultry and especially chicken do not require any special conditions for their breeding. Another advantage is their short reproductive cycle and incubation time of fertilized eggs. Nowadays chickens are breed mostly for meat. There are a lot of varieties and breeds which are classified as heavy meat-type chickens. However, an increase of body mass could cause problems with natural breeding because roosters are too heavy and thus are not able to reproduce naturally (Burton et al. 2016). This is the reason why artificial insemination (AI) is so necessary in poultry reproduction (Iqbal et al., 2002). AI process in birds includes the transportation of semen into female reproductive tract manually. It starts from the collection of the semen from the male and its evaluation in terms of motility, viability and concentration. The main advantage of AI in poultry is that one sexually mature male could cover up 20 females, which is important for an increased reproduction speed (Karayat et al., 2016). An important aspect of successful AI is the highest possible quality of fresh or cryopreserved sample. Nevertheless, commonly used cryoprotectants or semen extenders have a contraceptive or toxic effect and increase the risk of oxidative stress (OS) development associated with the overproduction of reactive oxygen species (ROS), (Çifti and Aygün, 2018). OS is one of the prime mediators responsible for reduction of ejaculate quality leading to malfunction of cell-egg interactions and male sub-fertility or infertility. OS is characterized as physiological imbalance between prooxidants and antioxidants. Under physiological conditions ROS are necessary for the sperm maturation, hyperactivation, capacitation, acrosome reaction as well as fertilization (Hubert et al., 2007; Mora et al., 2017). Avian spermatozoa are susceptible to oxidative damage due to high proportions of polyunsaturated fatty acids (PUFAs), a unique sperm head shape and membrane fluidity. PUFAs can be easily oxidized by ROS and make sperm cells more vulnerable to OS. In the intensive poultry production, animals are often exposed to negative conditions such as inadequate diet supplementation, fast growth, antibiotics or the other environmental stressors like atmospheric temperature, humidity, radiation, ammonia, bacterial and viral infections. All these conditions can stimulate ROS generation and enhance OS (Panda and Cherian, 2014; Surai, 2020). Although ROS production is involved in many biological functions of spermatozoa and excess of ROS could cause wide spectrum of negative effects such as decrease of sperm motility or membrane and acrosome damage. For the treatment of this condition compounds of natural origin with antioxidant activity are often used (Khan, 2011; Dutta et al., 2019). The addition of antioxidant compounds into fresh semen could defeat the deleterious effects of ROS on the sperm quality and fertility potential. Many antioxidants of natural origin provided positive effects on some sperm parameters such as motility and membrane integrity (Mehdipour et al., 2019). Curcumin (CUR) is a natural phenol produced by Curcuma longa characterized by bright yellow pigment and is commonly used as a spice or food-coloring agent. Therapeutic properties of curcumin are often associated with antioxidant and anti-inflammatory activity. Epicatechin (EPI) is a type of flavonoid compound very abundant in green tea, grape, cocoa bean and berries to which are ascribed a potential antioxidant activity (Omur and Coyan, 2016; Bernatoniene and Kopustinskiene, 2018). In our study were selected CUR and EPI because of high levels of their antioxidant activity. Previous reports studying the ROS-scavenging potential effects of CUR and EPI on mammalian spermatozoa (Tvrdá et al., 2016; Duračka et al., 2017; Greifová et al., 2017) showed promising results, however data in the field of avian reproduction are still missing. Therefore, it is necessary to find solutions for the potential application and use of CUR and EPI for the improvement of the quality of avian spermatozoa which are used for AI The objective of our study was to assess the effects of two selected antioxidants (CUR and EPI) on the selected quality parameters (motility, membrane and acrosome integrity) of rooster spermatozoa.

\section{MATERIAL AND METHODS}

\section{Sample collection and cultivation}

Sexually mature (61-67 weeks old) and clinically healthy Lohmann Light breeder males $(\mathrm{n}=10)$ reared in a private breeding facility (Liaharenský podnik Nitra Ltd., Slovak Republic) were used in the experiments. The roosters were housed in individual cages, under a constant photoperiod of $14 \mathrm{~h}$ of light day and were feed 
a commercial standard diet with water given ad libitum. Semen samples were obtained by massaging of cloaca, diluted in phosphate buffer saline (PBS, SigmaAldrich, St. Louis, USA) and cultured with different concentrations of CUR and EPI $(1,5,10,25,50$ and $100 \mu \mathrm{mol} / \mathrm{L}$ ). Selected quality parameters (motility, membrane and acrosome integrity) were analyzed after 0,2 and $24 \mathrm{~h}$ of in vitro culture at $4^{\circ} \mathrm{C}$

\section{Analysis of sperm motility}

For the evaluation of the sperm motility rate (\%, MOT), we used the computeraided sperm analysis (CASA, Version 14.0 TOX IVOS II., Hamilton-Thorne Bosciences, Beverly, MA, USA). All samples (10 $\mu \mathrm{l})$ were applied in Makler's counting chamber (depth $10 \mu \mathrm{m}, 37^{\circ} \mathrm{C}$; Sefi Medical Instruments, Haifa, Israel) and analyzed immediately.

\section{Membrane integrity}

Condition of the cell membrane was detected using a combination of eosin and nigrosin dye. Eosin is mainly used to stain the cytoplasm and cytoplasmatic membrane while nigrosin is used as a contrast dye for a better differentiation of the background. The slides for the determination of the cell membrane integrity were prepared as follows: we applied a drop of sperm suspension on the slide and stained it first with $4 \mu \mathrm{l}$ of eosin solution (Sigma-Aldrich, St. Louis, USA). Subsequently we added $4 \mu \mathrm{l}$ of nigrosin solution (Sigma-Aldrich, St. Louis USA). After the application of both dyes, we used another slide glass to prepare a smear. The samples were air-dried at a laboratory temperature and analyzed using a light microscope (Olympus, Tokyo, Japan) at a magnification of $40 \mathrm{x}$. On each slide we observed 100 cells to assess the percentage of live and dead cells.

\section{Acrosome integrity}

For the assessment of the acrosome integrity status, we used a double fast greenrose bengal stain technique. First of all, we applied $10 \mu \mathrm{l}$ of sample on a glass slide and we added the same volume of fast green-rose bengal mixture (SigmaAldrich, St. Louis, USA). The stained samples were incubated for 60 seconds and following incubation we used another glass slide to prepare a smear which was air-dried at laboratory temperature. All slides were analyzed using a light microscope (Olympus, Tokyo, Japan), evaluating the integrity and compactness of the sperm acrosome. Damaged acrosome was characterized by a disruption of the membrane and cluster stain present in the sperm head. At least 100 cells were observed in each slide and we calculated the percentage of cells with a damaged or normal acrosome.

\section{Statistical analysis}

All data were statistically evaluated using GraphPad Prism (version 6.0 for Windows, GraphPad Software incorporated San Diego, California, USA, http://www.graphpad.com/). Differences between control and experimental groups were statistically evaluated using one-way ANOVA analysis of variance followed by the Dunnet comparison test, comparing all groups amongst each other. The results obtained from the experimental groups are expressed as percentage of the control ( \pm S.D.). The statistical significance was set at the levels **** $(\mathrm{P}<0.0001)$; ***( $(\mathrm{P}<0.001) ; * *(\mathrm{P}<0.01) ; *(\mathrm{P}<0.05)$.

\section{RESULTS AND DISCUSSION}

\section{Evaluation of the sperm motility}

The results displayed in Figure 1 indicate that at time $0 \mathrm{~h}$, the sperm motility remained without any changes. Significant changes $(\mathrm{P}<0.001 ; \mathrm{P}<0.0001)$ were observed in the case of 50 and $100 \mu \mathrm{mol} / \mathrm{L}$ of CUR after $2 \mathrm{~h}$ of in vitro culture. The highest CUR concentrations decreased the sperm motility when compared to the control group. In contrast with CUR, in the case of EPI no differences were observed after $2 \mathrm{~h}$ of in vitro culture. Following $24 \mathrm{~h}$ of in vitro culture were recorded a significant $(\mathrm{P}<0.0001)$ preservation of the sperm motility in the case of both antioxidants at $1,5,10 \mu \mathrm{mol} / \mathrm{L}$ of CUR and $5,10,25,50 \mu \mathrm{mol} / \mathrm{L}$ of EPI. Contrary to our results Marouei et al. (2018) observed beneficial effects of higher CUR concentrations (200 and $300 \mu \mathrm{mol} / \mathrm{L}$ ) on rooster sperm motility parameters, viability and membrane integrity. However, the differences maybe explained by a different sample processing. Marouei et al. (2018) used postthawed semen samples, we used fresh samples of rooster sperm. In the study of Tvrdá et al. (2016) authors used CUR against oxidative stress developed using FeAA (ferrous ascorbate) in bull spermatozoa. The doses of CUR in a concentration range of $25-50 \mu \mathrm{mol} / \mathrm{L}$ lead to a preservation of sperm motility $(\mathrm{P}<0.001)$ when compared to the control group. We may hypothesize that the application of CUR and its effects depend on the species. In our study the CUR concentration of $50 \mu \mathrm{mol} / \mathrm{L}$ had the opposite effect and decreased the motility of rooster spermatozoa in comparison to the control group. These findings could support the theory that lower doses between 1-10 $\mu \mathrm{mol} / \mathrm{L}$ of CUR could exhibit higher preservation effects on fresh avian ejaculates. Kazemizadeh et al. (2019) focused on the effects of CUR under in vivo conditions. CUR was supplemented to the feed of twenty-eight broiler roosters at a concentration range of 10-30 $\mathrm{mg} /$ rooster per day from 61 weeks of age. Their study highlights the beneficial effects of increasing dietary supplementation of CUR on the sperm motility and plasma membrane integrity. Moreover, the authors used semen samples for artificial insemination and their results showed an improved $(\mathrm{P}<0.01)$ fertility rate in the groups supplemented with CUR in comparison with the control group. Al-Daraji (2012) studied the beneficial effects of grape flavonoids after in vitro supplementation on diluted rooster semen with $4 \mathrm{ml}$ of grape juice concentrate as a source of EPI. Their results revealed that following 1, 7 and 14 days of in vitro storage in the refrigerator (temperature $4-6{ }^{\circ} \mathrm{C}$ ) the presence of EPI significantly preserved the sperm motility $(\mathrm{P}<0.05)$ and improved percentage of live spermatozoa against the control group. Positive effects of EPI were also confirmed by Abdo et al. (2010). They added 1, 3 and 5\% green tea extract as a source of EPI into the diet of 21 Inshas roosters. The results of this study showed an improvement of the sperm concentration and a decreased percentage of damaged and dead sperm. Taking advantage of rabbit spermatozoa Ďuračka $\boldsymbol{e t}$ al. (2017) recorded positive and beneficial effects of CUR on one hand but also toxic and negative effect of this molecule on the motion behaviour. Similarly, to our report, higher doses (50 and $100 \mu \mathrm{mol} / \mathrm{L})$ of CUR significantly decreased $(\mathrm{P}<0.001)$ the percentage $(\%)$ of rabbit sperm motility when compared to the control group. On the other hand, higher doses $(100 \mu \mathrm{mol} / \mathrm{L})$ of EPI exhibited significant $(\mathrm{P}<0.001)$ preservation effects on the motility of rabbit spermatozoa.
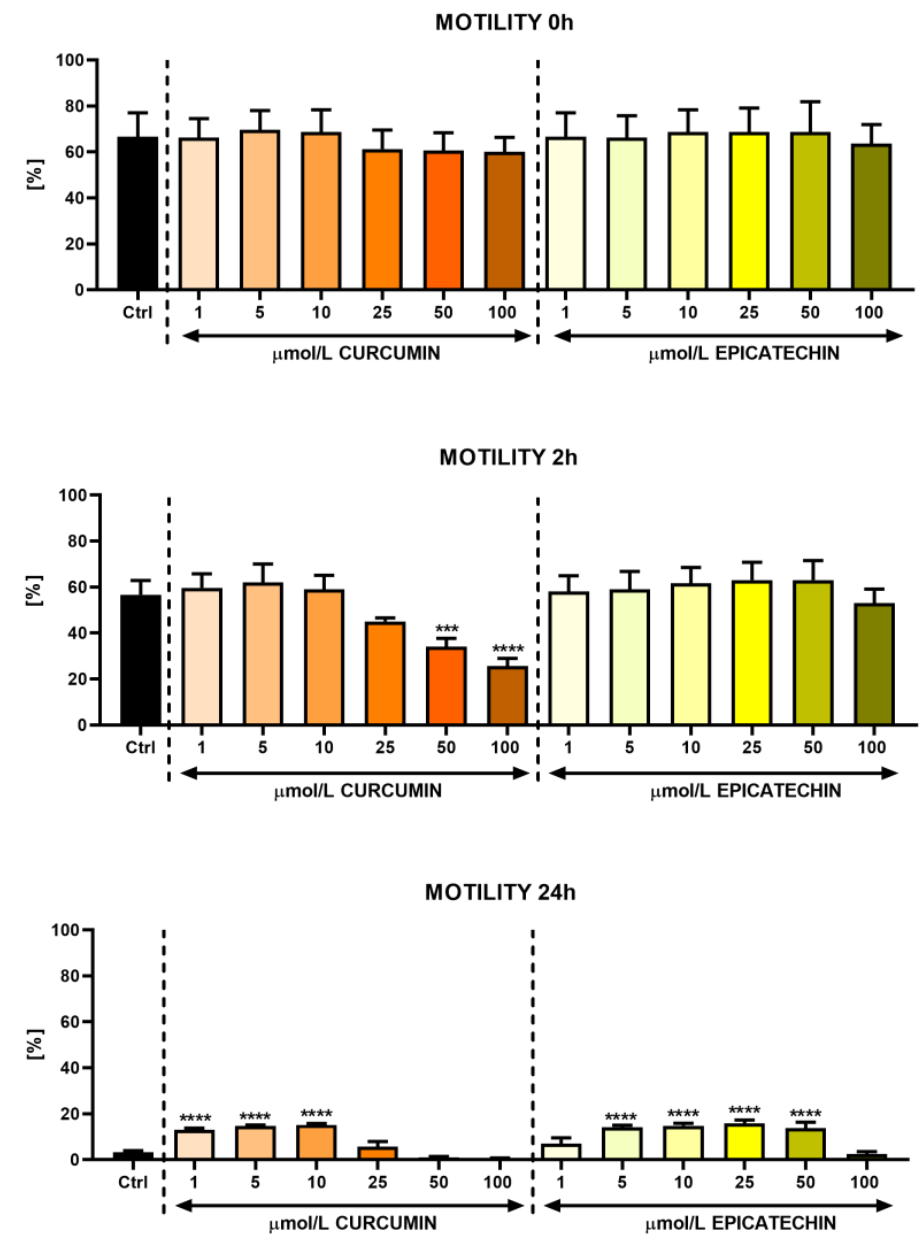

Figure 1 The effects of different concentrations of curcumin - CUR and epicatechin - EPI $(1,5,10,25,50$ and $100 \mu \mathrm{mol} / \mathrm{L})$ on the motility of rooster spermatozoa after 0,2 and $24 \mathrm{~h}$ of in vitro culture $(* * * \mathrm{P}<0.001 ; * * * * \mathrm{P}<0.0001)$.

\section{Membrane integrity}

In the case of the membrane integrity (Figure 2 ) no changes were noticed at time $0 \mathrm{~h}$ of the in vitro culture. After $2 \mathrm{~h}$ of incubation, the percentage of cells with damaged membrane was significantly decreased $(\mathrm{P}<0.01 ; \mathrm{P}<0.001)$ in the 
experimental groups exposed to 5 and $10 \mu \mathrm{mol} / \mathrm{L}$ CUR respectively. However, in the case of 50 and $100 \mu \mathrm{mol} / \mathrm{L}$ CUR were observed that the percentage of cells with damaged membranes was significantly higher $(\mathrm{P}<0.0001)$ when compared to the control group. This finding could support our hypothesis that high doses of CUR could exhibit toxic effects on the cells instead of antioxidant actions and damage the cell membrane even more. This phenomenon was even more pronounced after $24 \mathrm{~h}$ of in vitro culture. Inversely lower doses (5 and 10 $\mu \mathrm{mol} / \mathrm{L}$ ) of CUR showed protective effects while higher CUR concentrations (50 and $100 \mu \mathrm{mol} / \mathrm{L}$ ) had a negative impact on the cell membrane integrity. On the other hand, EPI exhibited positive effects up to $24 \mathrm{~h}$ of in vitro culture. There was a significant decrease $(\mathrm{P}<0.05 ; \mathrm{P}<0.01)$ of cells with damaged membrane in case of 25 and $50 \mu \mathrm{mol} / \mathrm{L}$ EPI. According to Yan et al. (2017) in vivo CUR supplementation exhibited positive effects and decreased the percentage of cells with damaged membranes while at the same time it increased the viability of rooster spermatozoa. Jalili et al. (2020) observed beneficial effects of CUR on the sperm quality parameters and fertility of broiler roosters. After 5 weeks of feeding roosters with different concentrations (0-30 mg/rooster/day) of CUR the function and integrity of the sperm plasma membrane was significantly increased $(\mathrm{P}<0.05)$ in all CUR treated groups. These observations could be compared with different effects of CUR under in vivo and in vitro conditions. It appears that higher doses of CUR exhibited better functions under in vivo conditions and during long term supplementation as a food additive.

\section{MEMBRANE INTEGRITY Oh}

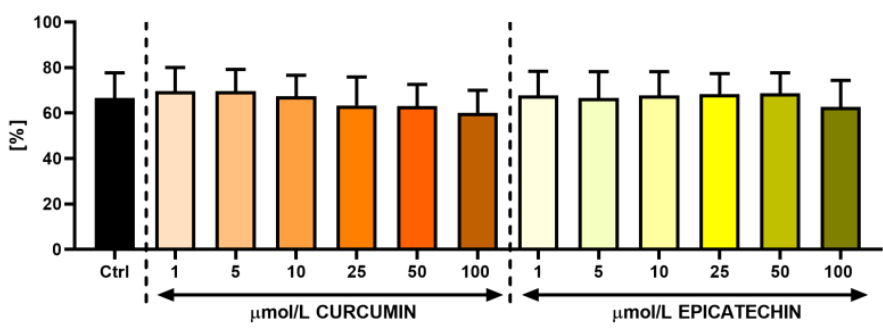

MEMBRANE INTEGRITY $2 \mathrm{~h}$

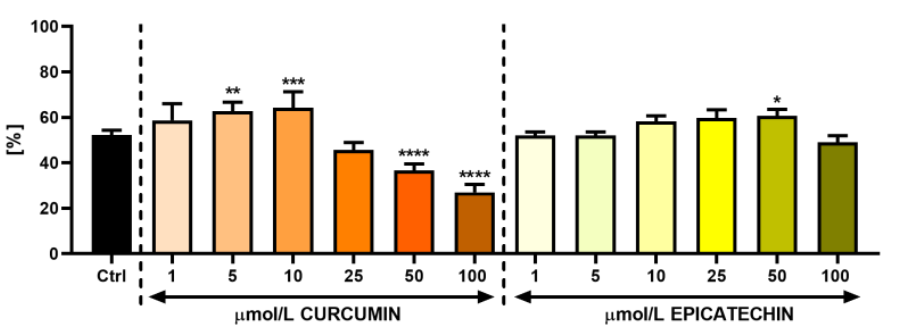

MEMBRANE INTEGRITY 24h

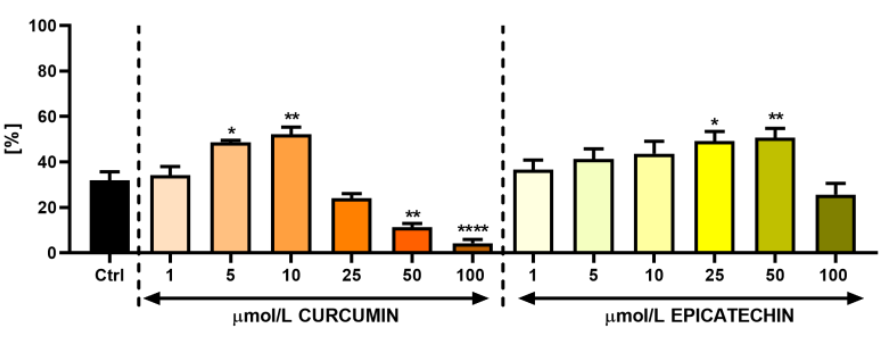

Figure 2 Membrane integrity of rooster spermatozoa after 0, 2 and $24 \mathrm{~h}$ in vitro culture with different concentrations of curcumin - CUR and epicatechin - EPI (1, $5, \quad 10, \quad 25, \quad 50$ and $100 \mu \mathrm{mol} / \mathrm{L}), \quad(* \mathrm{P}<0.05 ; \quad * * \mathrm{P}<0.01 ; \quad * * * \mathrm{P}<0.001$; $* * * * \mathrm{P}<0.0001)$.

In the already mentioned research of Kazemizadeh et al. (2019) CUR administration helped to increase the percentage of cells with a functional plasma membrane and to decrease the cell membrane damage in all groups with CUR supplementation. Greifová et al. (2017) evaluated the dose and time-dependent effects of EPI on bovine spermatozoa after 0,2, 6 and $24 \mathrm{~h}$ of in vitro culture. Similarly, to our research, the concentration of $50 \mu \mathrm{mol} / \mathrm{L}$ of EPI showed significant beneficial changes $(\mathrm{P}<0.001)$ in the motility and viability of cells after $24 \mathrm{~h}$ of in vitro culture. If we would compare the doses of CUR and EPI, we could see the differences between them. In most cases CUR exhibited better effects in lower doses but under in vitro conditions. Under in vivo conditions CUR displayed better antioxidant effects in higher doses. On the other hand, EPI functioned better in higher doses and after a longer exposure time.

\section{Acrosome integrity}

According to the assessment of the acrosome integrity (Figure 3) no significant differences were observed after 0 and $2 \mathrm{~h}$ of in vitro culture. Changes were visible after $24 \mathrm{~h}$ of incubation. As in the previous parameters CUR showed dual effects on the integrity of the acrosome. In case of 50 and $100 \mu \mathrm{mol} / \mathrm{L} \mathrm{CUR}$ were noticed a significant decrease $(\mathrm{P}<0.0001)$ of acrosome integrity when compared to the control group without CUR. Only $10 \mu \mathrm{mol} / \mathrm{L}$ CUR led to a significant increase $(\mathrm{P}<0.01)$ of the acrosome integrity and exhibited protective effects against oxidative stress-associated damage to the acrosome structures. Similarly, EPI displayed dichotomic effects on the acrosome integrity, as $50 \mu \mathrm{mol} / \mathrm{L} \mathrm{EPI}$ significantly increased $(\mathrm{P}<0.05)$ the level of acrosome integrity while $100 \mu \mathrm{mol} / \mathrm{L}$ significantly decreased $(\mathrm{P}<0.01)$ the percentage of cells with a compact acrosome. Raheja et al. (2018) used $1.5 \mathrm{mM}$ CUR extract as a supplement to the semen extender for the protection of bovine spermatozoa. Addition of CUR increased the progressive motility, plasma membrane integrity and percentage of cells with intact acrosomes. Chanapiwat and Kaeoket (2015) confirmed that lower doses of CUR exhibited better effects and increased the progressive motility, viability and acrosome integrity of cryopreserved boar semen. Omur $\boldsymbol{e}$ al. (2016) found out that antioxidants could be used as additives to freezing extenders and improve the quality of freeze thawed spermatozoa.
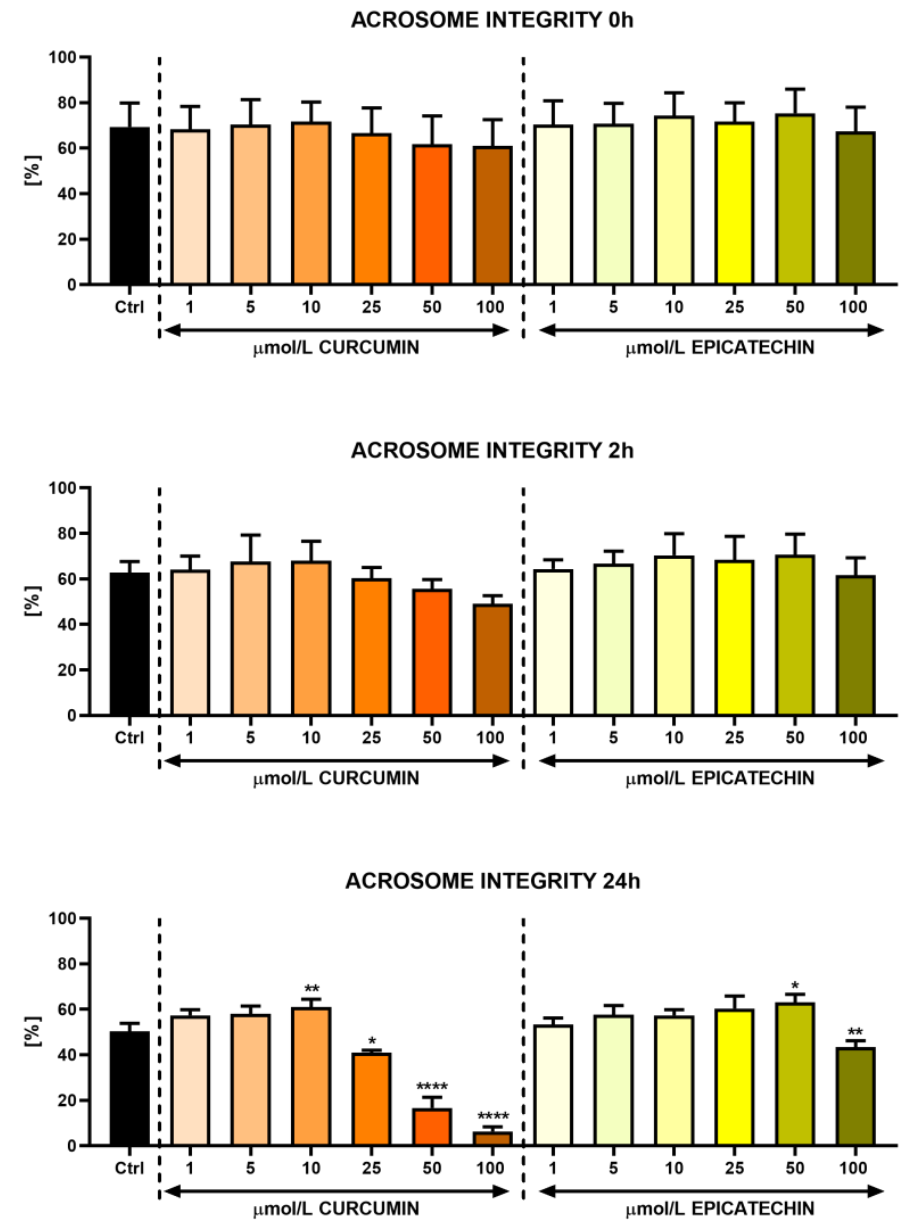

Figure 3 Acrosome integrity of rooster spermatozoa after 0,2 and $24 \mathrm{~h}$ of in vitro culture with different concentrations of curcumin - CUR and epicatechin - EPI (1, $5,10,25,50$ and $100 \mu \mathrm{mol} / \mathrm{L}),(* \mathrm{P}<0.05 ; * * \mathrm{P}<0.01 ; * * * * \mathrm{P}<0.0001)$.

They compared all groups supplemented with 1- and 2-mM CUR to the control group. Their results indicate a significant increase $(\mathrm{P}<0.05)$ of the percentage of sperm acrosome integrity. The addition of catechin to an extender for boar spermatozoa preserved the sperm motility, viability and decreased the acrosome damage caused by oxidative stress (Boonsorn et al., 2010). The use of higher doses of catechin $(50$ and $100 \mu \mathrm{mol} / \mathrm{L})$ provided outstanding results in all quality parameters of goat spermatozoa including the improvement of acrosome integrity 
of sperm cells exposed to catechin in comparison to the control group (Silva et al., 2019).

\section{CONCLUSION}

Based on our results the addition of antioxidants such as CUR and EPI could have positive as well as negative effects on rooster spermatozoa. The mechanism of their effects depends on numerous factors like dosage, animal species, in vitro/in vivo conditions and time interval of cultivation. Our experiments confirmed the beneficial and protective effects of both antioxidants against oxidative stress and defined their optimal doses for future experimental studies on avian spermatozoa.

Acknowledgments: This study was supported the APVV-15-0544 and VEGA $1 / 0239 / 20$ grants.

\section{REFERENCES}

Abdo, Z., Hassan, R. A., El-Salam, A. A., \& Helmy, S. A. (2010) Effect of adding green tea and its aqueous extract as natural antioxidants to laying hen diet on productive, reproductive performance and egg quality during storage and its content of cholesterol. Egyptian Poultry Science, 30(4), 1121-1149.

Al-Daraji H. J. (2012) The Use of Certain Grape Constituents for improve Semen Quality and Storage Ability of Diluted Roosters Semen. American Journal of Pharmtech Research, 2(5), 308-322.

Bernatoniene, J., \& Kopustinskiene D. M. (2018) The Role of Catechins in Cellular Responses to Oxidative Stress. Molecules: A Journal of Synthetic Chemistry and Natural Product Chemistry, 23(4), 965 https://doi.org/10.3390/molecules23040965

Boonsorn, T., Kongbuntad, W., Narkkong, N. A., \& Aengwanich, W. (2010) Effects of Catechin Addition to Extender on Sperm Quality and Lipid Peroxidation in Boar Semen. American-Eurasian Journal of Sustainable Agriculture, 7(3), 283-288.

Burton, E., Gatcliffe, J., Masey O’Neill, H., \& Scholey, D. (2016). Sustainable Poultry Production in Europe. CABI.

Çifti, H. B., \& Aygün, A. (2018) Poultry Semen Cryopreservation Technologies. World's Poultry Science Journal, 74(4), 699-710. https://doi.org/10.1017/s0043933918000673

Dutta, S., Ahmad, M., \& Agarwal, A. (2019) Oxidative stress and sperm function: A systematic review on evaluation and management. Arab Journal of Urology, 17(2), 87-97. https://doi.org/10.1080/2090598X.2019.1599624

Ďuračka, M., Halenár, M., \& Tvrdá, E. (2017) In vitro effects of selected biologically active compounds on rabbit spermatozoa motility behaviour. Journal of Microbiology, Biotechnology and Food Sciences, 6(6), 1290-1294. https://doi.org/10.15414/jmbfs.2017.6.6.1290-1294

Greifová, H., Tvrdá, E., Jambor, T., \& Lukáč, N. (2017) Dose- and timedependent effects of epicatechin on bovine spermatozoa in vitro. Journal of Microbiology, Biotechnology and Food Sciences, 7(3), 235-239. https://doi.org/10.15414/jmbfs.2017/18.7.3.235-239

Hubert, A. J., Pamplona, R., Buffenstein, R., \& Buttemer, W. A. (2007) Life and death: metabolic rate, membrane composition, and life span of animals.

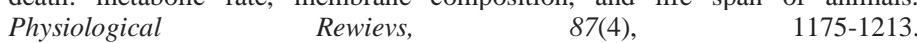
https://doi.org/10.1152/physrev.00047.2006

Chanapiwat, P., \& Kaeoket, K. (2015) The effects of Curcuma longa extracted (curcumin) on the quality of cryopreserved boar semen. Animal Science Journal, 86(9), 863-868. https://doi.org/10.1111/asj.12395

Iqbal, M., Cawthon, D., Wideman R. F., Beers, F., \& Bottje, W. G. (2002) Antioxidant enzymes activities, and mitochondrial fatty acids in pulmonary hypertension syndrome (PHS) in broilers. Poultry Science, 81(2), 252-260. https://doi.org/10.1093/ps/81.2.252

Jalili, F., Zareh-Shahneh, A., Zeinoaldini, S., Yousefi, A. R., \& Kazemidazeh, A. (2020) The effect of curcumin on frozen-thawed sperm quality and fertility of broiler breeder roosters. Iranian Journal of Animal Science, 50(4), 295-306.

Kazemizadeh, A., Zare Shahneh, A., Zeinoaldini, S., Yousefi, A. R., Mehrabani Yeganeh, H., Ansari Pirsaraei, Z., \& Akhlaghi, A. (2019) Effects of dietary curcumin supplementation on seminal quality indices and fertility rate in broiler breeder roosters. British Poultry Science, 60(3), 256-264. https://doi.org/10.1080/00071668.2019.1571165

Khan, R. (2011) Antioxidant and poultry semen quality. World's Poultry Science Journal, 67(2), 297-308. https://doi.org/10.1017/S0043933911000316

Kharayat, N. S., Chaudhary G. R., Katiyar R., Balmurugan, B., Patel, M., Uniyal, S., Raza, M., \& Mishra G. K. (2016) Significance of Artificial Insemination in Poultry. Journal of Veterinary Science and Technology, 5(1), 1-5. https://doi.org/10.37591/rrjovst.v5i1.519

Marouei, T., Daghigh Kia H., \& Moghaddam G. A. (2018) Effects of different levels of Curcumin antioxidant in Beltsville modified diluent on rooster sperm quality after freeze-thawing process. Journal of Animal Science Researches, 28(1), 65-76.

Mehdipour, M., Daghigh Kia, H., \& Najafi A. (2019) Effect of crocin and naringenin supplementation in cryopreservation medium on post-thawed rooster sperm quality and expression of apoptosis associated genes. bioRxiv. https://doi.org/10.1101/846758

Mora, A. R., Firth, A., Blareau, S., Vallat, A., \& Helfenstein, F. (2017) Oxidative stress affects sperm performance and ejaculate redox status in subordinate house sparrows. Journal of Experimantal Biology, 220(14), 2577-2588 https://doi.org/10.1242/jeb.154799

Omur, A. D., \& Coyan K. (2016) Protective effects of the antioxidant's curcumin, ellagic acid and methionine on mitility, mitochondrial transmembrane potential, plasma membrane and acrosome integrity in freeze-thawed Merino ram sperm. Veterinarní Medicína, 61(1), 10-16. https://doi.org/10.17221/8677. VETMED

Panda, A. K., \& Cherian, G. (2014) Role of Vitamin E in Counteracting Oxidative Stress in Poultry. The Journal of Poultry Science, 51(2), 109-117. https://doi.org/10.2141/jpsa.0130134

Raheja, N., Choudhary, S., Grewal, S., Sharma N., \& Kumar, N. (2018) A review on semen extenders and additives used in cattle and buffalo bull semen preservation. Journal of Entomology and Zoology Studies. 6(3), 239-245.

Silva, E. C. B., Arruda, L. C. P., Vieira, J. I. T., Soares, P. C., \& Guerra, M. M. P. (2019) (+)-Catechin and (-)-epigallocatechin gallate: are these promising antioxidant therapies for frozen goat semen? Arquivo Brasileiro de Medicina Veterinária e Zootecnia, 71(2), 521-528. https://doi.org/10.1590/1678-4162$\underline{\mathbf{1 0 5 3 9}}$

Surai, P. F. (2020) Antioxidants in Poultry Nutrition and Reproduction: An Update. Antioxidants, 9(2), 105. https://doi.org/10.3390/antiox9020105

Tvrdá, E., Tušimová, E., Kováčik, A., Páal, D., Greifová, H., Abdramanov, A., \& Lukáč, N. (2016) Curcumin has protective and antioxidant properties on bull spermatozoa subjected to induced oxidative stress. Animal Reproduction Science, 172, 10-20. https://doi.org/10.1016/j.anireprosci.2016.06.008

Yan, W., Kanno, Ch., Oshima, E., Kuzuma, Y., Kim, S. W., Bai, H., Takahashi, M., Yanagawa, Y., Wakamatsu J., \& Kawahara, M. (2017) Enhancement of sperm motility and viability by turmeric by-product dietary supplementation in roosters. Animal Reproduction Science, 185, 195-204. https://doi.org/10.1016/j.anireprosci.2017.08.021 